\title{
Predictors of AVP and TSH Levels and the Timing of First Voiding in the Newborn
}

\author{
TUOMO VUOHELAINEN, RIITTA OJALA, ANITA VIRTANEN, JARI LAATTA, PERTTI MÖRSKY, JUKKA UOTILA, \\ AND OUTI TAMMELA
}

\begin{abstract}
Paediatric Research Centre [T.V., R.O., A.V., J.L., O.T.], University of Tampere and Tampere University Hospital, FIN 33014 Tampere, Finland; Department of Pediatrics [R.O., O.T.], Centre for Laboratory Medicine [P.M.], Department of Obstetrics and Gynecology [J.U.], Tampere University Hospital, FIN 33521 Tampere, Finland
\end{abstract}

\begin{abstract}
To evaluate obstetric predictors of umbilical cord plasma AVP levels, serum TSH levels and the timing of first voiding, 87 singleton term newborns were divided into three groups: group A, vaginal delivery $(n=30)$; group $\mathrm{B}$, cesarean section (CS) during labor $(n=26)$; and group C, elective CS $(n=31)$. The AVP concentration was $120(0.7-2170) \mathrm{ng} / \mathrm{L}$ in group A, $1.8(0.01-183)$ $\mathrm{ng} / \mathrm{L}$ in group $\mathrm{B}$, and $0.8(0.01-30) \mathrm{ng} / \mathrm{L}$ in group $\mathrm{C}(p<0.001)$. In group A, the TSH concentration was 10.20 (3.5-30.80) $\mathrm{mU} / \mathrm{L}$; in group B, $5.40(2.10-43.00) \mathrm{mU} / \mathrm{L}$; and in group C, $5.30(2.90-$ $11.00) \mathrm{mU} / \mathrm{L}(p=0.001)$. Duration of labor had a positive correlation with $\operatorname{AVP}(p<0.001)$ and TSH $(p=0.001)$ concentrations. The timing of first voiding had a positive correlation with gestational age ( $p=0.003$ ), volume of additional feeding before first voiding ( $p<$ $0.001)$, and umbilical AVP concentration $(p=0.023)$. The AVP and TSH concentrations are associated with mode of delivery and duration of labor and AVP levels also with the timing of first voiding in the newborn. (Pediatr Res 62: 106-110, 2007)
\end{abstract}

$\mathrm{D}$ elay of neonates' first voiding beyond $24 \mathrm{~h}$ often attracts special attention from the nursing staff and causes parental anxiety. It has previously been concluded that micturition delayed over $24 \mathrm{~h}$ is usually a benign phenomenon and maturation of the infant, duration of labor, and use of paracervical block predict this in healthy neonates (1). However, the factors affecting the timing of the newborn infant's first voiding remain unclear.

AVP is a peptide hormone released into the circulation from the neurohypophysis. At low physiologic blood concentrations, AVP induces mainly antidiuretic effects, and at high blood concentrations, e.g. in shock states, AVP has a powerful vasoconstrictive effect, although its importance in healthy states is minor. When the body is low on water, AVP causes the kidneys to conserve water by concentrating the urine and reducing urine volume.

After normal vaginal delivery, the umbilical cord plasma AVP concentrations of newborns are higher than after CS (2-5). The precise reasons for and role of high AVP concentrations in response to labor and birth remain elusive, and currently there are no adequate explanations for the phenomenon. Intrauterine stress, hypoxia, or possibly asphyxia may induce AVP secretion by an unknown mech-

Received July 26, 2006; accepted February 24, 2007.

Correspondence: Outi Tammela, M.D., Ph.D., Tampere University Hospital, P.O. Box 2000, FIN 33521 Tampere, Finland; e-mail: outi.tammela@uta.fi anism $(2,6,7)$. There are also indications that cranial mechanical compression in the birth canal might constitute a reason (2). AVP is probably an essential factor in neonatal adaptation to living in the extrauterine environment. It induces a redistribution of fetal blood flow from peripheral areas to vital organs (8).

Another hypophyseal peptide hormone, TSH or thyroidstimulating hormone, is measured from the umbilical cord serum at birth as a routine screening for congenital hypothyroidism in every newborn in Finland (9). Fetal stress and hypoxia evidently increase the umbilical TSH concentration (10-13). As with AVP, the mode of delivery and various unfavorable antepartum conditions appear to increase the umbilical blood TSH level at birth. Again, the precise mechanism and purpose of an increased umbilical TSH concentration in response to stress remain unclear.

In the present study, we sought to test the hypothesis that high fetal AVP concentrations and delayed voiding are linked with stressful birth. We hypothesized that high fetal AVP concentrations are associated with later voiding in newborn infants. To the best of our knowledge, this has not previously been studied. Because of the routine screening of TSH, we decided to also add this factor to our analysis. Our hypothesis was that the changes in TSH levels and AVP levels behave in a similar way, both being associated with the mode of delivery and with a stressful birth. We also intended to establish whether labor pain experienced by mothers have an influence on infants' umbilical AVP and TSH concentrations or time of the first voiding.

\section{SUBJECTS AND METHODS}

The present investigation was carried out at Tampere University Hospital in January 2001 and May 2002. The ethical committee of Tampere University Hospital approved the study protocol and informed consent was obtained from the mothers participating. According to our preliminary power analysis, a sufficient statistical significance level of $p<0.05$ and a statistical power of $80 \%$ were achieved with 16 infants per group as based on our earlier results on delayed micturition in healthy neonates (1). In this earlier study, $2.9 \%$ of infants had micturition delayed over $24 \mathrm{~h}$ and the difference between the mean time of micturition in infants born by elective CS as against vaginal delivery was at least 1 SD. Our initial goal was therefore to recruit 20 infants for each group.
Abbreviations: AVP, arginine vasopressin; CS, cesarean section; ICP, intracranial pressure; TSH, thyrotropin; VAS, visual analog scale 
The study population comprised 87 normal term infants born between $37-42 \mathrm{wk}$ of gestation. The subjects were divided into three subgroups according to mode of delivery: group A, vaginal delivery $(n=30)$; group B, CS after onset of labor $(n=26)$; and group C, elective CS $(n=31)$. There were no emergency sections. In group A, 4 infants were delivered with vacuum extraction, 1 with forceps delivery, and the remaining 25 by normal vaginal delivery. In group B, there was only one case in which labor had progressed to the second stage; in the other cases, the decision on CS was made during the first stage. Twins and triplets, infants $<37 \mathrm{wk}$ of gestation, and neonates with major malformations were excluded from the study.

Details of delivery, including duration of labor, umbilical cord length, umbilical arterial $\mathrm{pH}$, and mother's blood loss, and of infants' birth characteristics, including gestational age, birth weight, and Apgar score were collected from birth records. The data on infants' health after birth, the time of the first micturition, and daily weight were recorded in the newborns' case records by the midwife and the personnel on the maternity ward. The time of the first voiding was established by visual observation. Micturition was classified as delayed if the first voiding occurred later than $24 \mathrm{~h}$ after birth. The details of the antenatal data and infants' condition after birth are presented in Table 1 .

Immediately after birth, $3 \mathrm{~mL}$ of blood was drawn from the umbilical cord for the measurement of plasma AVP concentration. The specimens were collected into ice-chilled tubes containing $\mathrm{Na}_{2}$ EDTA and taken to the laboratory. Plasma was separated in a refrigerated centrifuge, frozen, and sent to United Laboratories Ltd., Helsinki, for the assay of AVP. The cord plasma AVP concentration was measured by using an AVP RIA Mitsubishi kit (Mitsubishi Ltd., Yuka, Japan).

Determination of TSH in umbilical cord serum was performed at our laboratory by time-resolved fluoroimmunoassay. The assay was run in an AutoDELFIA analyzer using AutoDELFIA hTSH Ultra B042-201 reagents (PerkinElmer Life and Analytical Sciences Wallac Ltd, Turku, Finland).

On the day after delivery, mothers in group A and B were asked to fill in a VAS questionnaire to evaluate the subjective intensity of labor pain. The scale ranged between 0 and 5, 0 indicating mild pain and 5 severe pain. Mothers' subjective opinion about adequacy of the received pain relief was also inquired in questionnaire.

Table 1. Antenatal data and data on infants' condition after birth

\begin{tabular}{|c|c|c|c|}
\hline & $\begin{array}{l}\text { Group A } \\
(n=30)\end{array}$ & $\begin{array}{l}\text { Group B } \\
(n=26)\end{array}$ & $\begin{array}{l}\text { Group C } \\
(n=31)\end{array}$ \\
\hline Primipara mother & $13(43.3)$ & $17(65.4)$ & $10(32.3)$ \\
\hline $\begin{array}{l}\text { Time of amniotomy (h) } \\
\text { Duration of }\end{array}$ & $3.1(0.2-58.5)$ & $8.1(0-31.5)$ & $0(0-9.7)$ \\
\hline Pregnancy (wk) & $40.1(0.9)$ & $40.3(1.2)$ & $39.0(0.7)$ \\
\hline First stage of labor (h) & $7.2(1.1-19.5)$ & $0.2(0.05-0.4)$ & - \\
\hline $\begin{array}{l}\text { Second stage of labor } \\
\text { (min) }\end{array}$ & $10(5-61)$ & $1.3(0-1.3)$ & \\
\hline Labor (total) (h) & $7.5(1.4-20.7)$ & $12.7(2.3-26.8)$ & \\
\hline $\begin{array}{l}\text { Apgar }<7 \text { at } 1 \mathrm{~min} \text { of } \\
\text { age }\end{array}$ & $2(6.7)$ & $2(7.7)$ & $2(6.5)$ \\
\hline $\begin{array}{l}\text { Apgar }<7 \text { at } 5 \mathrm{~min} \text { of } \\
\text { age }\end{array}$ & 0 & 0 & 0 \\
\hline Umbilical artery pH & $7.24(0.09)$ & $7.31(0.05)$ & $7.32(0.04)$ \\
\hline $\begin{array}{l}\text { Length of umbilical cord } \\
(\mathrm{cm})\end{array}$ & $63(45-90)$ & $58(43-83)$ & $53(33-70)$ \\
\hline Infants weight $(\mathrm{g})$ & 3667 (490) & $3643(420)$ & 3795 (539) \\
\hline $\begin{array}{l}\text { Breast feeding in the } \\
\text { delivery room }\end{array}$ & $29(96.7)$ & $17(65.4)$ & $24(77.4)$ \\
\hline $\begin{array}{l}\text { Additional feeding } \\
\text { before first miction }\end{array}$ & $4(13.3)$ & $13(50)$ & $8(25.8)$ \\
\hline $\begin{array}{l}\text { Volume of additional } \\
\text { milk (mL) }\end{array}$ & $0(0-185)$ & $2.5(0-125)$ & $0(0-90)$ \\
\hline $\begin{array}{l}\text { Maximum weight loss } \\
\text { during hospitalization } \\
(\%)\end{array}$ & $5.8(1.8-9.4)$ & $6.3(0.8-10.8)$ & $7.3(1.4-9.5)$ \\
\hline $\begin{array}{l}\text { Age of maximum weight } \\
\text { loss (d) }\end{array}$ & $2.4(0.8)$ & $2.7(0.9)$ & $2.8(1.0)$ \\
\hline $\begin{array}{l}\text { Age at hospital } \\
\text { discharge }(\mathrm{d})\end{array}$ & $4(1-8)$ & $5(4-8)$ & $5(3-15)$ \\
\hline
\end{tabular}

Values are expressed as occurrences (\%), mean (SD), or median (range) values.
Statistical analyses were made using SPSS for Windows version 10.1. Continuous data were analyzed using Spearman's rank correlation, MannWhitney $U$ test, or Kruskal-Wallis test, and discrete data were analyzed by the Pearson $\chi^{2}$ test or Fisher's exact test. Factors influencing the incidence of newborns' delayed voiding in the whole population were analyzed with backward-stepwise logistic regression analysis, with $p<0.05$ and 95\% confidence intervals $(\mathrm{CI})$ to indicate statistical significance. In this analysis, the time of micturition was classified as delayed after $12.83 \mathrm{~h}$, which constitutes the upper quartile. Covariates included in the model were mode of delivery, gestational age, time from membrane rupture, AVP level, TSH level, birth weight, umbilical cord length, volume of additional feeds besides mother's breast feeding before first voiding, pain relief during labor, and epidural analgesia.

\section{RESULTS}

The umbilical AVP concentration differed significantly between the groups studied. The highest concentration was in infants delivered vaginally (group A) and lowest in infants delivered by elective CS (group C). In group A, the AVP concentration was $120(0.7-2170) \mathrm{ng} / \mathrm{L}$; in group $\mathrm{B}, 1.8$ $(0.01-183) \mathrm{ng} / \mathrm{L}$; and in group C, $0.8(0.01-30) \mathrm{ng} / \mathrm{L}(p<$ $0.001)$. AVP concentrations between groups A versus B $(p<$ $0.001)$ and A versus $\mathrm{C}(p<0.001)$ were statistically significant. By contrast, between groups $\mathrm{B}$ and $\mathrm{C}$ there was no statistical difference in AVP levels $(p=0.100)$.

There was a significant positive correlation between umbilical AVP concentration and duration of the first stage of labor $(r=0.644, p<0.001)$, umbilical cord length $(r=0.333, p=$ $0.002)$, and gestational age $(r=0.240, p=0.025)$. There was also a trend toward negative correlation between maximum weight loss and umbilical AVP concentration $(r=-0.206$, $p=0.055)$. In contrast, medical pain relief, the use of epidural analgesia, the mothers' subjective experience of labor pain, the duration of the second stage of labor or infants' birth weight did not influence AVP concentration.

There was a significant positive correlation between AVP and TSH concentrations $(r=0.340, p=0.001)$. The mode of delivery had an effect on TSH concentration very similar to that on AVP. In group A, the TSH concentration was 10.20 (3.5-30.80) $\mathrm{mU} / \mathrm{L}$; in group B, $5.40(2.10-43.00) \mathrm{mU} / \mathrm{L}$; and in group C, $5.30(2.90-11.00) \mathrm{mU} / \mathrm{L}(p=0.001)$. TSH concentrations between vaginally delivered and both CS groups were statistically significant: A versus $\mathrm{B}(p=0.003)$ and A versus $\mathrm{C}(p=0.001)$. As earlier with AVP, between groups $\mathrm{B}$ and $\mathrm{C}$ there was no statistical difference in TSH levels. Again, as with AVP, there was a significant positive correlation between umbilical cord serum TSH concentration and the duration of the first stage of labor $(r=0.451, p=$ 0.001 ).

All infants voided spontaneously. The first micturition took place at $8.7(0-38.9)$ hours of age in group A, $7.8(0-31.0)$ hours in group B, and $0.8(0-16.5)$ hours in group C $(p=$ $0.017)$. The timing of first micturition between vaginally delivered and CS infants was not statistically significant. However, there was a significant difference between groups $\mathrm{A}$ versus $\mathrm{C}(p=0.013)$ and B versus $\mathrm{C}(p=0.015)$. Delayed micturition was detected in altogether four $(4.6 \%)$ infants, three in group A and one in group B. All these 4 and $31(40 \%)$ of the remaining infants defecated in the delivery room $(p=$ 0.031 ) and their birth weight was lower; in corresponding 
order, 3065 (3000-3570) g versus $3720(2700-5430) \mathrm{g}(p=$ 0.011) compared with the remaining infants. A significant positive correlation was observed between time of first micturition and gestational age $(r=0.313, p=0.003)$, volume of extra feeds in addition to mother's breast feeding before first voiding $(r=0.529, p<0.001)$, and umbilical AVP concentration $(r=0.243, p=0.023)$. There was no correlation between the time of the infants' first micturition and medical pain relief, the use of epidural analgesia, mothers' subjective assessment of intensity of labor pain, umbilical blood TSH concentration, duration of labor or the second stage of labor, infants' condition after birth, infants' birth weight, or infants' maximum weight loss. Out of the covariates tested in the logistic regression analysis, a higher gestational age $[p=$ 0.020; odds ratio (OR), 1.975; CI, 1.115-3.500] and volume of additional feeding ( $p=0.028$; OR, 1.031; CI, 1.003-1.059) predicted statistically significantly delayed voiding beyond the upper quartile $(12.83 \mathrm{~h})$. Same multivariate analysis was attempted within each of three major groups, but there were no statistically significant findings.

Twenty-seven (90\%) mothers in group A and $22(85 \%)$ in group B returned the pain questionnaire. Among respondent mothers, $19(70 \%)$ in group A and $19(86 \%)$ in group B felt they had received adequate pain relief in due course (NS). Eight mothers in group A and three in group B were dissatisfied with the pain relief given during labor. Among these unsatisfied mothers, two persons in group A and one in group B had in fact received no medical pain relief at all. The subjectively assessed intensity of labor pain was 3.40 (0.90$4.90)$ in group A and $3.68(0-4.95)$ in group B (NS). There was no connection between the mode of pain relief and the subjective intensity of labor.

\section{DISCUSSION}

Fetal plasma AVP concentration in the umbilical cord has been shown to be increased after vaginal delivery. Also, in infants delivered by CS after the onset of labor, the umbilical cord AVP blood concentration has been noted to be higher than in infants delivered by elective CS (3). In our current study, we also found the highest umbilical AVP correlations in the vaginally delivered group. However, between the two CS groups, the umbilical AVP concentrations did not differ statistically significantly. The results show that a long duration of overall labor and especially the duration of the first stage of labor are associated with the increased umbilical AVP concentration in newborn infants. On the other hand, we found no such correlation between AVP concentration and the duration of the second stage of labor as previously reported (4).

The mechanism of fetal AVP release has been thought to be an effect of the reduction in placental blood flow during uterine contraction, causing acute fetal stress and hypoxia and elevated intracranial pressure $(2,5,7)$. The negative correlation between umbilical $\mathrm{pH}$ and AVP concentration supports the concept of acute stress affecting the fetal AVP release. It is also possible that the more mature the infant is, the more probable will be the placental insufficiency causing fetal hypoxia and raising the AVP concentration, although a previous small study found no connection between AVP concentration and gestational age (14). Also, Hadeed and associates (2) reported no difference in AVP concentrations between term and preterm infants. Interestingly, there was no association here between elevated AVP concentration and birth weight.

As previously noted, mothers who had an emergency CS were excluded. There was also no association between umbilical cord length and mode of delivery. The significant positive correlation between the newborn's AVP concentration and umbilical cord length is probably associated with an increased risk of umbilical cord compression and hence a greater risk of hypoxia with a longer cord. This would also support the theory of stress being at least one of the factors behind AVP secretion. The correlation of AVP concentration with the duration of the first stage of labor would also corroborate such a conception. The AVP concentration is elevated when the duration of labor and therefore the amount of stress is greater.

What is the specific stimulus that causes this peak in fetal AVP concentration during labor? Interestingly, Hadeed speculated that head compression and elevated ICP might be the reason for the increased AVP secretion during a normal vaginal birth. According to Hadeed, AVP concentrations were significantly lower in a group consisting of breech-delivered infants (2). As discussed in above, AVP raises blood pressure at high concentrations. During labor and contractions when the ICP is elevated, it would be consistent for AVP to be secreted in an effort to raise blood pressure and ensure cerebral perfusion pressure. It has previously been shown that there is an association between increased ICP and AVP release (15). In addition, AVP may have a protective role during hypoxia, and increased ICP may by a negative feedback mechanism reduce the blood flow to the choroid plexus and the production of cerebrospinal fluid (16).

In contrast to the results of a recent study, we found no correlation between infants' acidosis or and umbilical TSH level (13). This was probably because our study population was relatively small. On the other hand, we found a positive correlation between the duration of the first stage of labor and elevated TSH concentration, this giving support to earlier reports (11). The TSH level would thus appear to reflect fetal stress. Vaginal delivery raised the infants' umbilical TSH level significantly. The mode of delivery has been shown to be the strongest independent factor underlying increased umbilical TSH concentration (11). The strong association between umbilical blood AVP and TSH concentrations favors the conception of global hypophyseal hormone hypersecretion in response either to fetal stress or to head compression during vaginal delivery.

It has previously been noted that $93-100 \%$ of full-term infants pass urine within $24 \mathrm{~h}$ of birth $(1,17,18)$. Delay of the first micturition over $24 \mathrm{~h}$ of age is usually a benign phenomenon connected with functional hypoperfusion of the infants' kidneys and poor fluid intake during the first days of life $(19,20)$. Also, the maturation of the infant, the duration of labor, and the use of paracervical block were associated 
with delayed micturition in healthy neonates (1). In the current study, we noted first voiding beyond $24 \mathrm{~h}$ of life in $4.6 \%$ of infants, which is in accord with the incidence previously reported. The small number of such cases in this study made the analysis of these infants as a group of their own not possible. Because of this limitation, we chose the upper quartile of timing of first void to identify potential predictors for delayed micturition with logistic regression analysis.

To the best of our knowledge, the correlation between AVP and first micturition has not previously been studied. Here, an increase in umbilical AVP concentration was associated with later voiding in newborn infants. It is conceivable that birth stress induces an increase in fetal AVP secretion and causes hemodynamic adaptation with a redistribution of fetal blood flow from peripheral areas to vital organs (8). This peak increase in fetal AVP concentration would thus be associated with or perhaps cause the delayed voiding seen in newborns. In our study, delayed voiding seemed to be strongly associated with elevated AVP. Another explanation for this phenomenon might be the mode of delivery, but multivariate analysis did not give any support for this or AVP. Therefore, there is only evidence of associations, and our data are insufficient to show any causality. Greater gestational age also correlated with delayed voiding. As speculated above, this phenomenon might be explained by hypoxia due to placental insufficiency associated with more mature infants.

Plasma AVP levels fell to normal within the first days of life $(2,3,14)$, which may explain why first voiding was delayed over $24 \mathrm{~h}$ so infrequently, as well as the absent connection between first voiding and weight loss. We found a trend toward a negative correlation between maximum weight loss and umbilical AVP concentration. As expected, there was no connection between elevated umbilical TSH and delayed micturition.

The more additional feeding the infant received, the later the first micturition took place. In our hospital, own mother's milk is substituted in full-term infants only if the infant's condition requires it and breast milk is not secreted sufficiently. Personnel on the maternity ward follow the time of infants' first voiding, and if an infant does not pass urine they may be more prone to give additional milk. The prevailing practice in the hospital may thus explain the association between delayed first micturition and additional feeding.

In labor, the pain accompanying uterine contractions provokes a generalized neuroendocrinal stress response including increased oxygen consumption, hyperventilation, metabolic acidemia, effects of the hypophyseal-pituitary axis, including the stimulation of AVP release, and increased autonomic activity leading to increased catecholamine release and decreased placental perfusion. In consequence of these responses, fetal acidosis may develop (21). Pharmacologic pain relief during labor can reduce these responses, but it can also, on the other hand, cause fetal side-effects. Epidural analgesia may cause maternal hypotension and longer duration of the second stage of labor exposing the fetus to acidemia and hypoxia (22-24).

In two earlier studies, paracervical block has been seen to cause fetal bradycardia and be associated with the newborn's first micturition delayed for longer than $24 \mathrm{~h}(1,25)$. In this study, mothers were relatively satisfied with the pain relief given during the delivery. The mode of pain relief did not affect the subjective intensity of labor pain. Nor was there any correlation between the time of first micturition, umbilical AVP or TSH concentrations, and mother's subjective experiences. Likewise, pharmacological pain relief did not affect the time of first urination, umbilical blood AVP, or TSH levels.

We conclude that there is an obvious association between vaginal delivery and elevated AVP and TSH concentrations such as has been shown in earlier studies. There is also an association between high AVP concentrations and delayed voiding. On the other hand, labor pain experienced by the mother did not affect either the newborns' AVP or TSH concentrations or the time of first micturition, which bespeaks the fact that the fetus and the mother are separate individuals each having protective mechanisms of their own to survive birth and delivery.

Our study gives support to earlier findings indicating that hypoxia and stress increase infant's AVP and TSH secretion during birth. There are probably combinations of factors contributing to this elevation in AVP and TSH concentrations, one being head compression and increased ICP itself.

Further studies are needed to investigate the etiology and mechanism of increased AVP and TSH concentrations during birth.

\section{REFERENCES}

1. Tammela OK, Ikonen S, Nieminen P, Tammela TL 1997 Delayed first voiding in healthy neonates and obstetric analgesia. Prenat Neonatal Med 2:263267

2. Hadeed AJ, Leake RD, Weitzman RE, Fisher DA 1979 Possible mechanism of high blood levels of vasopressin during the neonatal period. J Pediatr 94:805808

3. Pohjavuori M, Fyhrquist F 1980 Hemodynamic significance of vasopressin in the newborn infant. J Pediatr 97:462-465

4. Pohjavuori M 1983 Obstetric determinants of plasma vasopressin concentrations and renin activity at birth. J Pediatr 103:966-968

5. Pohjavuori M, Raivio K 1985 The effects of acute and chronic perinatal stress on plasma vasopressin concentration and renin activity at birth. Biol Neonate 47:259264

6. Speer ME, Gorman WA, Kaplan SL, Rudolph AJ 1984 Elevation of plasma arginine vasopressin following perinatal asphyxia. Acta Paediatr Scand 73:610 614

7. Ramin SM, Porter JC, Gilstrap LC, Rosenfeld CR 1991 Stress hormones and acid-base status of human fetuses at delivery. J Clin Endocrinol Metab 73:182186

8. Iwamoto HS, Rudolph AM, Keil LC, Heymann MA 1979 Hemodynamic responses of the sheep fetus to vasopressin infusion. Circ Res 44:430-436

9. Virtanen M, Mäenpää J, Pikkarainen J, Pitkänen L, Perheentupa J 1989 Aetiology of congenital hypothyroidism in Finland. Acta Paediatr Scand 78:67-73

10. Lao TT, Li CY, Panesar NS 1992 Transient neonatal hyperthyrotropinaemia. Early Hum Dev 28:19-25

11. Chan LY, Leung TN, Lau TK 2001 Influence of perinatal factors on cord blood thyroid-stimulating hormone level. Acta Obstet Gynecol Scand 80:10141018

12. Chan LY, Chiu P, Lau TK 2003 Cord blood thyroid-stimulating hormone level in high-risk pregnancies. Eur J Obstet Gynecol Reprod Biol 108:142-145

13. Chan LY, Fok WY, Sahota D, Lau TK 2006 Cord blood thyroid-stimulating hormone level and risk of acidosis at birth. Eur J Obstet Gynecol Reprod Biol 124:173-177

14. Rees L, Forsling ML, Brook CG 1980 Vasopressin concentrations in the neonatal period. Clin Endocrinol (Oxf) 12:357-362 
15. Gaufin L, Skowsky W, Goodman S 1977 Release of antidiuretic hormone during mass-induced elevation of intracranial pressure. J Neurosurg 46:627-637

16. Faraci FM, Kinzenbaw D, Heistad DD 1994 Effect of endogenous vasopressin on blood flow to choroid plexus during hypoxia and intracranial hypertension. Am J Physiol 266:H393-H398

17. Sherry SN, Kramer I 1955 The time of passage of the first stool and urine by the newborn infant. J Pediatr 46:158-159

18. Clark DA 1977 Times of first void and first stool in 500 newborns. Pediatrics 60:457-459

19. Moore ES, Galvez MB 1972 Delayed micturition in the newborn period. J Pediatr 80:867-873

20. Wang PA, Huang FY 1994 Time of the first defaecation and urination in very low birth weight infants. Eur J Pediatr 153:279-283
21. Brownridge P 1995 The nature and consequences of childbirth pain. Eur J Obstet Gynecol Reprod Biol 59:S9-S15

22. Grenman S, Erkkola R, Kanto J, Scheinin M, Viinamäki O, Lindberg R 1986 Epidural and paracervical blockades in obstetrics. Acta Obstet Gynecol Scand 65:699-704

23. Scherer R, Holzgreve W 1995 Influence of epidural analgesia on fetal and neonatal well-being. Eur J Obstet Gynecol Reprod Biol 59:S17-S29

24. Lieberman E, O'Donoghue C 2002 Unintended effects of epidural analgesia during labor: a systematic review. Am J Obstet Gynecol 186:S31-S68

25. Palomäki O, Huhtala H, Kirkinen P 2005 A comparative study of the safety of $0.25 \%$ levobupivacaine and $0.25 \%$ racemic bupivacaine for paracervical block in the first stage of labor. Acta Obstet Gynecol Scand 84:956-961 